\title{
Onset of Convection in Strongly Shaken Granular Matter
}

\author{
Peter Eshuis, ${ }^{1}$ Devaraj van der Meer, ${ }^{1}$ Meheboob Alam, ${ }^{2}$ Henk Jan van Gerner, ${ }^{1}$ Ko van der Weele, ${ }^{3}$ and Detlef Lohse ${ }^{1}$ \\ ${ }^{1}$ Physics of Fluids Group, Department of Science and Technology, J. M. Burgers Center, and Impact and Mesa+ Institutes, \\ University of Twente, P.O. Box 217, 7500 AE Enschede, The Netherlands \\ ${ }^{2}$ Engineering Mechanics Unit, Jawaharlal Nehru Center for Advanced Scientific Research, Jakkur P.O. Bangalore 560064, India \\ ${ }^{3}$ Department of Mathematics, Division of Applied Analysis, University of Patras, 26500 Patras, Greece
}

(Received 2 April 2009; published 22 January 2010)

\begin{abstract}
Strongly vertically shaken granular matter can display a density inversion: A high-density cluster of beads is elevated by a dilute gaslike layer of fast beads underneath ("granular Leidenfrost effect"). For even stronger shaking the granular Leidenfrost state becomes unstable and granular convection rolls emerge. This transition resembles the classical onset of convection in fluid heated from below at some critical Rayleigh number. The same transition is seen in molecular dynamics (MD) simulations of the shaken granular material. The critical shaking strength for the onset of granular convection can be calculated from a linear stability analysis of a hydrodynamiclike model of the granular flow. Experiment, MD simulations, and theory quantitatively agree.
\end{abstract}

DOI: 10.1103/PhysRevLett.104.038001

PACS numbers: 45.70.Qj, 05.65.+b, 47.20.Bp

The holy grail question in research on granular dynamics is [1,2], To what extent can granular flow be described by a continuum approach? Though such hydrodynamic equations can formally be derived from basic principles of statistical physics (i.e., the Boltzmann equations with the Chapman-Enskog kinetic theory [2-6]), it is questionable whether they correctly describe granular flow, as the separation of the microscopic length and time scales from the macroscopic ones is much less pronounced than one would desire for a continuum description [2] and clustering of the grains [7] due to the inelastic nature of their collision plays a dominant role. Indeed, explicit counterexamples have been found due to an inelastic collapse [8], though in one dimension [9] and thus somehow artificial. Nonetheless, in many special situations the static (i.e., time-independent) hydrodynamic equations [10-12] for granular flow successfully describe the observed phenomena, in principle allowing for their application in various fields such as process technology or geophysics.

In this Letter we analyze strongly vertically shaken granular matter, which can display a density inversion with a high-density cluster of slow beads being elevated by a dilute gaslike layer of fast beads underneath. This phenomenon has been dubbed granular Leidenfrost effect [13], in analogy to the classical Leidenfrost effect displayed by a water droplet on a hot plate, elevated by its own vapor. Indeed, the density profiles as function of the shaking strength can be calculated $[13,14]$ from the timeindependent hydrodynamiclike equations [10] and show very good agreement with the experimental results [13].

However, as experimentally observed, for even stronger shaking $\left[\Gamma=a(2 \pi f)^{2} / g\right.$ typically $50-80$ for the dimensionless acceleration, where $a$ and $f$ are shaking amplitude and frequency, and $g$ gravitational acceleration] and large enough aspect ratio the inverted density profile becomes unstable and convection rolls develop, both in 3D [15] and
quasi-2D [16]. Similar rolls were obtained in molecular dynamics (MD) simulations [17,18]. Note that these convection rolls are of very different nature than those at much less strongly ( $\Gamma$ typically $3-10)$ shaken granular matter [19]. Those rolls emerge from the interaction of the grains with the surrounding walls and are thus a boundary effect [20] and not a bulk instability as in the present case of strongly shaken granular matter.

Here the convection rolls resemble the classical Rayleigh-Bénard (RB) convection rolls emerging in fluid heated from below and cooled from above $[21,22]$ beyond a critical temperature difference, or, when nondimensionalized, beyond a critical Rayleigh number $\mathrm{Ra}_{c}$, which can analytically be calculated from a linear stability analysis of the underlying continuum equations [21] (the NavierStokes equations and the thermal advection equation in the Boussinesq approximation). The famous result for an infinite aspect ratio sample is $\mathrm{Ra}_{c} \approx 1101$ (with open top boundaries) and $\mathrm{Ra}_{c} \approx 1708$ (with rigid top boundaries) [23].

The key question is, Can one follow the analogous approach for the time-dependent continuum equations of granular flow and obtain the critical shaking strength from the linear stability analysis of the Leidenfrost state? In other words, is the description of granular flow with time-independent continuum equations extendable to the time-dependent case? Such a linear stability analysis has already been performed [24] for chute flows, where the formation of longitudinal vortices was predicted, but no quantitative comparison with experiments or numerical simulations could be done. Another example is the linear stability analysis of a system of inelastically colliding hard spheres, driven by two opposite "thermal" walls [25], but again there was no comparison with experiment. Further examples for linear stability analyses of granular continuum equations include that for a thin layer of shaken 
granular matter [26] and closest to the system here that for vertical shaken granular matter in a closed box [27].

In this Letter we will positively answer the above key question, at least in respect to the calculation of the onset of convection: We will first show our experimental results on the onset of convection in strongly shaken granular matter, then we will present the results from molecular dynamics simulations, which quantitatively resemble the experimental ones. The main result of this Letter is the theoretical calculation of the onset of convection by performing a linear stability analysis of the continuum equations of granular flow and, in particular, the favorable comparison with experiments and MD simulations.

Experiment.-Our experimental setup consists of a quasi-2D Perspex container of dimensions $L \times D \times H$ with an adjustable container length $L=10-202 \mathrm{~mm}$, a depth $D=5 \mathrm{~mm}$, and a height $H=150 \mathrm{~mm}$. The container is partially filled with steel beads of diameter $d=$ $1.0 \mathrm{~mm}$, density $\rho=7800 \mathrm{~kg} / \mathrm{m}^{3}$, and coefficient of normal restitution $e \approx 0.9$. The setup is mounted on a sinusoidally vibrating shaker with tunable frequency $f$ and amplitude $a$. The experiments are recorded with a highspeed camera at a frame rate of 1000 frames per second.

The appropriate dimensionless control parameters to analyze the experiments in this strong shaking regime are $[13,16]$ (i) the dimensionless shaking strength [28]

$$
S=\frac{a^{2}(2 \pi f)^{2}}{g d},
$$

which is the ratio of the kinetic energy inserted into the system by the vibrating bottom and the potential energy associated with the particle diameter $d$; (ii) the number $F$ of bead layers, defined as $F \equiv N_{p} d^{2} /(L D)$, where $N_{p}$ is the number of particles (determined from the total mass); (iii) the inelasticity parameter $\varepsilon=\left(1-e^{2}\right)$; and (iv) the length ratio $L / d$.

At strong shaking strength $S$ and large enough bed height (i.e., large enough $F$ ), the density-inverted Leidenfrost state is formed [13], in which a cluster of slow, almost immobile particles is supported by a gaseous region of fast particles underneath. When further increasing $S$, eventually a number of particles becomes more mobile (higher granular temperature) than the surrounding ones and creates an opening in the floating cluster of the Leidenfrost state, as seen in Fig. 1. These particles have picked up an excess of energy from the vibrating bottom (due to a statistical fluctuation) and collectively move upwards, very much like a hot fluid parcel at the bottom plate in Rayleigh-Bénard convection. This upward motion of the highly mobile beads must be balanced by a downward movement of neighboring particles, leading to the formation of a pair of convection rolls. Since the downward motion is most easily accomplished at the sidewalls (due to the extra source of dissipation, i.e., the friction with the walls), the first convection roll always forms near a sidewall. As shown in Fig. 1, within a second this first roll triggers the formation of rolls along the entire length of the

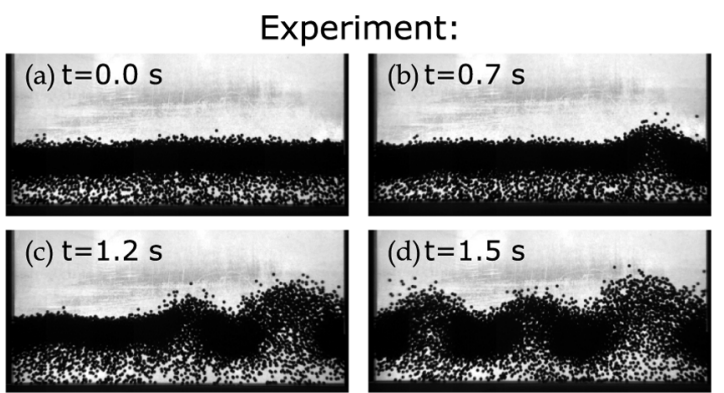

MD simulation:
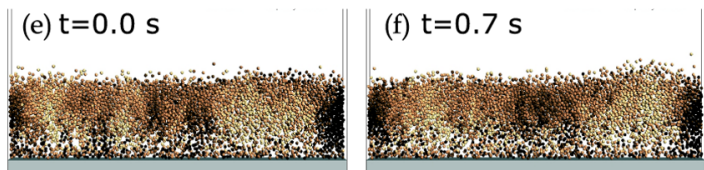

(g) $\mathrm{t}=1.2 \mathrm{~s}$

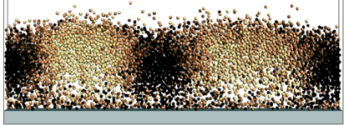

(h) $\mathrm{t}=1.5 \mathrm{~s}$

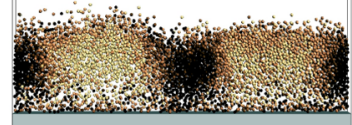

FIG. 1 (color online). Onset of convection in experiment and in MD simulations. (a)-(d) Snapshots of $F=11.1$ layers of $d=$ $1.0 \mathrm{~mm}$ steel beads in a container of length $L / d=101$ shaken at an amplitude of $a=3.0 \mathrm{~mm}$. The frequency was linearly increased from $f=42 \mathrm{~Hz}$ to $f=48 \mathrm{~Hz}$ at a rate of $90 \mathrm{~Hz} / \mathrm{min}$. The transition from the steady Leidenfrost state to fully developed convection took place at $f=45 \mathrm{~Hz}$ [between frames (a) and (b)]. (e)-(h) Snapshot from the corresponding MD simulations. Again, $F=11.1$, shaking amplitude $a=3.0 \mathrm{~mm}$, and linearly increased frequency (at the rate of $90 \mathrm{~Hz} / \mathrm{min}$ ) with $f=44 \mathrm{~Hz}$ in (e). The onset of convection takes place at a frequency of $f=45 \mathrm{~Hz}(S=75$, frame $f)$. The light-colored particles are moving upward and the dark ones downward. Respective movies of both experiments and numerical simulations can be seen in Ref. [39].

container, leading to a fully developed convection pattern. Such experiments are repeated for various filling heights $F$. The respective critical shaking strength $S_{c}$ for the onset of convection is shown in Fig. 2; it strongly depends on $F$, roughly exponentially as $S_{c} \sim \exp (0.2 F)$.

Molecular dynamics simulations. - In order to investigate information not available in experiment, we have also performed molecular dynamics simulations, using a granular dynamics code $[29,30]$ to numerically study the shaken quasi-2D granular material. The MD code calculates the particle trajectories from Newton's equations of motion, with the particle-particle interactions being given by a 3D soft sphere collision model, including tangential friction $[29,30]$. We have used the same parameters and dimensions as in the equivalent experiment: The container is filled with $N_{p}=L / d \times D / d \times F$ identical spherical particles, i.e., the number which corresponds to the filling height $F$. The coefficient of restitution $e$ and friction coefficient for the particle-particle and particle-wall interactions determine the total energy dissipation in the system. The friction coefficient is set to 0.03 , while the coefficient of normal restitution (which for simplicity is 


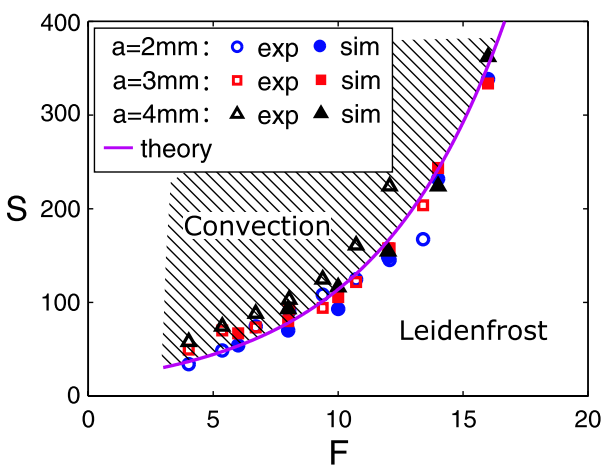

FIG. 2 (color online). Convection threshold in the $(S, F)$ phase diagram from experiments, MD simulations, and theory. The experiments and simulations are performed with $d=1 \mathrm{~mm}$ glass beads, with shaking amplitude $a=2.0 \mathrm{~mm}$ (blue dots), $a=3.0 \mathrm{~mm}$ (red squares), and $a=4.0 \mathrm{~mm}$ (black triangles). The shaking strength $S$ is increased by increasing the frequency $f$. The open symbols correspond to the onset of convection in the experiments and the solid ones to the onset in the MD simulations. The solid line is the result of the linear stability analysis of the Leidenfrost state $(\operatorname{Pr}=1.7)$.

assumed to be velocity-independent) is fitted to correctly describe the experimentally found onset for the case of $F=10$ layers, yielding the very realistic values of $e=$ 0.957 for steel and $e=0.905$ for glass beads for both particle-particle and particle-wall interactions.

The numerical results for $S_{c}(F)$ are shown in Fig. 2, too, and they well agree with the experimental results within numerical and experimental precision. Snapshots from the numerical simulations are shown in Fig. 1, again showing a one-to-one correspondence with the experiments.

Linear stability analysis.-Following [27], we will now calculate the experimentally and numerically found onset $S_{c}$ of convection from a linear stability analysis of the suggested $[10,13,14]$ (two-dimensional) hydrodynamical equations for the three fields of the granular flow, namely, the number density $n(x, y, t)$, the velocity $\mathbf{u}(x, y, t)=$ $\langle\mathbf{c}(x, y, t)\rangle \equiv\left(u_{x}(x, y, t), u_{y}(x, y, t)\right)$ of the granular flow, and the granular temperature $T(x, y, t)=\frac{1}{2} m\left\langle(c-\langle\mathbf{c}\rangle)^{2}\right\rangle$, which is defined as the fluctuation kinetic energy of the particles around their mean velocity, with the mass $m$ of a single particle, $\mathbf{c}$ the instantaneous particle velocity, and the angular brackets denoting a suitable averaging. The hydrodynamic equations we will employ here and which approximately describe the dynamics of these three fields are $[10,13,14]$ the continuity equation

$$
\frac{\partial n}{\partial t}+\boldsymbol{u} \cdot \boldsymbol{\nabla} n+n \boldsymbol{\nabla} \cdot \boldsymbol{u}=0,
$$

the momentum balance equation

$$
\begin{aligned}
m n\left(\frac{\partial \boldsymbol{u}}{\partial t}+\boldsymbol{u} \cdot \boldsymbol{\nabla} \boldsymbol{u}\right)= & m n \vec{g}-\boldsymbol{\nabla} p+\boldsymbol{\nabla} \cdot\left(\mu\left[\boldsymbol{\nabla} \boldsymbol{u}+(\boldsymbol{\nabla} \boldsymbol{u})^{T}\right]\right) \\
& +\boldsymbol{\nabla}(\lambda \boldsymbol{\nabla} \cdot \boldsymbol{u}),
\end{aligned}
$$

in which $p$ is the pressure, $\mu$ the shear viscosity, and $\lambda$ the second viscosity, and the granular energy balance equation

$$
n\left(\frac{\partial T}{\partial t}+\boldsymbol{u} \cdot \boldsymbol{\nabla} T\right)=\boldsymbol{\nabla} \cdot(\kappa \boldsymbol{\nabla} T)-p(\boldsymbol{\nabla} \cdot \boldsymbol{u})-I,
$$

where $\kappa$ is the analog of thermal conductivity for a granular gas and $I$ is the dissipative term due to the inelastic particle collisions [31]. The granular hydrodynamic equations (2)(4) are complemented by the respective global and local boundary conditions [particle conservation, extremum of $u_{x}(x, y, t)$ at the bottom of the container, $u_{y}(x, 0, t)=0$ at the bottom wall, vanishing velocities at the top $y \rightarrow \infty$, and finally temperature agreeing with the static temperature profile at the bottom and at the top $(y \rightarrow \infty)]$ and by the constitutive relations for the pressure field $p$, the energy dissipation rate $I$, and the transport coefficients $\kappa, \mu$, and $\lambda$. Since our system combines dilute, gaseous regions with clusters where the density approaches the close-packed value, we must take excluded volume effects into account [32]. Therefore, as equation of state for the twodimensional granular fluid we take $[10,13,14] p=$ $n T\left(n_{c}+n\right) /\left(n_{c}-n\right)$, with $n_{c}=2 / \sqrt{3} d^{2}$ the number density of a hexagonal close-packed crystal, accounting for the excluded area of the gas. The constitutive relation for the energy dissipation rate $I$ reads $[10,13,14] \quad I=$ $\varepsilon n T \sqrt{T / m} /\left(\gamma_{c} \ell\right)$. Here the inelasticity parameter $\varepsilon=$ $\left(1-e^{2}\right)$ naturally shows up in the theoretical model. The value for the constant $\gamma_{c}=2.26$ has been adopted from [10]. Also the parametrization of the thermal conductivity is taken from [10,14], namely, $\kappa=n(\alpha \ell+d)^{2} \sqrt{T / m} / \ell$, with the mean free path $\ell=\left(n_{c}-n\right) /\left[\sqrt{8} n d\left(n_{c}-a n\right)\right]$, the constants $a=1-\sqrt{3 / 8}$ and $\alpha=0.6$ [33]. For the granular shear viscosity $\mu$ various choices are proposed [34]. Here we express $\mu$ in terms of the Prandtl number Pr, namely, $\mu=m \operatorname{Pr} \kappa$. As $\operatorname{Pr}$ measures the ratio between convective and diffusive energy transfer, which should be roughly the same in the granular flow, we expect $\operatorname{Pr} \sim 1$. Finally, because the viscosity $\mu$ for our granular system behaves so analogously to classical fluids, we use the Stokes approximation to get $\lambda=-\mu$ for the second viscosity [34].

The linear stability procedure applied to the equations (2)-(4) is in principle analogous to the one used to determine the onset of Rayleigh-Bénard convection in classical fluids [21]; however, the base state is more complicated. Rather than linearizing around the linear-temperature and constant-density base state as in classical RB flow, we now linearize around the Leidenfrost state [13] with the dense and colder granular cluster on top of the gaseous and warmer region. These nontrivial height dependences of both granular temperature and density are numerically known from Ref. [13]. The base state does not display any lateral variation of the granular temperature or density and the granular velocity is zero. The linearization around the Leidenfrost base state leads to an eigenvalue problem, which is solved with the spectral-collocation method [3537] with periodic boundary conditions in the horizontal direction. The onset of convection corresponds to the in- 
stability of the Leidenfrost base state and thus to the occurrence of positive eigenvalues $\gamma>0$. The wave number $k_{x}$ corresponding to the most unstable mode (maximal $\gamma$ value) determines the dominant perturbation that leads to the transition from the Leidenfrost state to the convective state [38].

The excellent agreement of the results from the linear stability analysis with experiments and MD simulations is most impressively seen in Fig. 2, showing the onset of convection for various numbers $F$ of layers [39]. The only fit parameter we have used is the Prandtl number, the value of which is set to $\operatorname{Pr}=1.7$. The convective rolls predicted by our theory are stationary in nature and originate due to a pitchfork bifurcation from the granular Leidenfrost state. In the classical RB case, too, the first bifurcation from the conduction state is responsible for the genesis of stationary convection rolls at some critical Rayleigh number.

Conclusions. - We have shown that in strongly vertically shaken granular matter the experimentally and numerically observed phase transition from the density-inverted granular Leidenfrost state to the convective state can quantitatively be calculated from a linear stability analysis of the time-dependent continuum theory of granular flow. Of course, a full comparison goes beyond linear stability theory and requires the (numerical) solution of the full nonlinear partial differential equations. Future work will also have to reveal the sensitivity of the results to the employed equation of state, accurate constitutive relations [40], and transport coefficients in granular flow, which will allow us to determine them with considerably enhanced confidence.

We thank G. W. Bruggert, M. Bos, and R. Bos for help with the experiments, M. van der Hoef and H. Kuipers for making their code $[29,30]$ available to us, and S. Luding for discussions. This work is part of the research program of FOM, which is financially supported by NWO.

[1] H. M. Jaeger, S. R. Nagel, and R. P. Behringer, Rev. Mod. Phys. 68, 1259 (1996).

[2] I. Goldhirsch, Annu. Rev. Fluid Mech. 35, 267 (2003).

[3] I. Goldhirsch, S. Noskowicz, and O. Bar-Lev, Phys. Rev. Lett. 95, 068002 (2005).

[4] J. T. Jenkins and S. B. Savage, J. Fluid Mech. 130, 187 (1983); J. Jenkins and M. Richman, J. Fluid Mech. 171, 53 (1986); C. S. Campbell, Annu. Rev. Fluid Mech. 22, 57 (1990).

[5] N. Sela and I. Goldhirsch, J. Fluid Mech. 361, 41 (1998).

[6] J. J. Brey, J. W. Dufty, C. S. Kim, and A. Santos, Phys. Rev. E 58, 4638 (1998).

[7] I. Goldhirsch and G. Zanetti, Phys. Rev. Lett. 70, 1619 (1993).

[8] Y. Du, H. Li, and L. P. Kadanoff, Phys. Rev. Lett. 74, 1268 (1995).

[9] N. Sela and I. Goldhirsch, Phys. Fluids 7, 507 (1995).

[10] E. L. Grossman, T. Zhou, and E. Ben-Naim, Phys. Rev. E 55, 4200 (1997).

[11] I. S. Aranson and L. S. Tsimring, Rev. Mod. Phys. 78, 641 (2006).
[12] J. Duran, Sands, Powders, and Grains: An Introduction to the Physics of Granular Materials (Springer, New York, 2000).

[13] P. Eshuis, K. van der Weele, D. van der Meer, and D. Lohse, Phys. Rev. Lett. 95, 258001 (2005).

[14] B. Meerson, T. Pöschel, and Y. Bromberg, Phys. Rev. Lett. 91, 024301 (2003).

[15] R. D. Wildman, J. M. Huntley, and D. J. Parker, Phys. Rev. Lett. 86, 3304 (2001).

[16] P. Eshuis et al., Phys. Fluids 19, 123301 (2007).

[17] R. Ramírez, D. Risso, and P. Cordero, Phys. Rev. Lett. 85, 1230 (2000).

[18] D. Paolotti et al., Phys. Rev. E 69, 061304 (2004).

[19] E. E. Ehrichs et al., Science 267, 1632 (1995); J. B. Knight, H. M. Jaeger, and S. R. Nagel, Phys. Rev. Lett. 70, 3728 (1993).

[20] M. Bourzutschky and J. Miller, Phys. Rev. Lett. 74, 2216 (1995); S. Luding et al., Phys. Rev. E 50, R1762 (1994).

[21] S. Chandrasekhar, Hydrodynamic and Hydromagnetic Stability (Dover, New York, 1981).

[22] E. Bodenschatz, W. Pesch, and G. Ahlers, Annu. Rev. Fluid Mech. 32, 709 (2000).

[23] H. Jeffreys, Philos. Mag. 2, 833 (1926); Proc. R. Soc. A 118, 195 (1928).

[24] Y. Forterre and O. Pouliquen, J. Fluid Mech. 467, 361 (2002).

[25] E. Khain and B. Meerson, Europhys. Lett. 65, 193 (2004).

[26] C. Bizon, M. D. Shattuck, and J. B. Swift, Phys. Rev. E 60, 7210 (1999).

[27] E. Khain and B. Meerson, Phys. Rev. E 67, 021306 (2003).

[28] Note that it is not $\Gamma$-for reasons explained in [16]. Indeed, $S$ is obtained as natural dimensionless parameter when nondimensionalizing below Eqs. (2)-(4).

[29] M. A. van der Hoef et al., Adv. Chem. Eng. 31, 65 (2006).

[30] N. G. Deen et al., Chem. Eng. Sci. 62, 28 (2007).

[31] In Eq. (4) we did not include terms which are quadratic in $\boldsymbol{\nabla} \boldsymbol{u}$. For the heat flux we also neglected a small $[5,13]$ term proportional to the gradient of the number density.

[32] This is one of the major differences with the stability work of [27] which probes the onset of convection in the Boltzmann limit of a dilute granular gas. The other differences are the boundary conditions at the top and the Leidenfrost base state in the present case, with nonuniform density and nonlinear temperature profile.

[33] More accurate (and intricate) forms of the constitutive relations $[2,6]$ have been used in the literature which for granular gases normally give very similar results.

[34] J. J. Brey, M. J. Ruiz-Montero, and F. Moreno, Phys. Rev. E 63, 061305 (2001); X. He, B. Meerson, and G. Doolen, Phys. Rev. E 65, 030301 (2002); T. Ohtsuki and T. Ohsawa, J. Phys. Soc. Jpn. 72, 1963 (2003).

[35] S. A. Orszag, J. Fluid Mech. 50, 689 (1971).

[36] M. Alam and P. Nott, J. Fluid Mech. 377, 99 (1998); M. Alam et al., J. Fluid Mech. 523, 277 (2005).

[37] C. Canuto et al., Spectral Methods: Fundamentals in Single Domains (Springer, New York, 2006).

[38] The details of this procedure will be published elsewhere.

[39] See supplementary material at http://link.aps.org/ supplemental/10.1103/PhysRevLett.104.038001 for a comparison between theoretical and experimental velocity and density profiles.

[40] S. H. Noskowicz et al., Europhys. Lett. 79, 60001 (2007). 